\title{
A Hierarchical Deformable Model Using Statistical and Geometric Information
}

\author{
Dinggang Shen ${ }^{1}$ and Christos Davatzikos ${ }^{1,2,3}$ \\ ${ }^{1}$ Department of Radiology, ${ }^{2}$ Department of Computer Science, ${ }^{3}$ Center for Computer-Integrated \\ Surgical Systems and Technology, Johns Hopkins University \\ Emails:dgshen@cbmv.jhu.edu,hristos@parthenon.rad.jhu.edu
}

\begin{abstract}
A new deformable model has been proposed by employing a hierarchy of affine transformations and an adaptive-focus statistical model. An attribute vector is used to characterize the geometric structure in the vicinity of each point of the model; the deformable model then deforms in a way that seeks regions with the similar attribute vectors. This is in contrast to most active contour models, which deform to nearby edges without considering the geometric structure of the boundary around an edge point. Furthermore, a deformation mechanism that is robust to local minima is proposed, which is based on evaluating the snake energy function on segments of the snake at a time, instead of individual points. Various experimental results show the effectiveness of the proposed methodology.
\end{abstract}

\section{Introduction}

Deformable models have been used extensively in image analysis, especially in medical or biological imaging applications [1,2], where the objects (or structures) to be analyzed undergo deformations.

For applications in which a training set is available, statistical models, incorporating statistical knowledge about the object and its variability, have been proposed $[3,12]$. On the other hand, flexible Fourier contour and surface models [4], performing statistics on Fourier parameters [5], were applied into the segmentation of 2D and 3D objects from MRI volume data.

Hierarchical approaches usually increase the likelihood of finding the globally optimal match, where the calculations of finer details of the deformation are performed after matching on a more global scale. Multiscale techniques have been successful in improving the performance of the snake model $[6,11]$.

In this paper, we investigate a hierarchical method using a series of global and local affine transformations for approximating the actual deformation existing between the initially provided model and the desired object. The main characteristics of our model are the following:

(a) A model contour is first constructed from a training set. Attached to each point on the model are two kinds of information. First, an attribute vector, which reflects shape characteristics of the model around each point, from a local and finer scale to a more global and coarse scale. The attribute vectors are essential in our formulation, since they distinguish different parts of a boundary according to their shape properties. Second, statistical information about the expected shape variation, which is determined via a training set. Importantly, the statistical information is expressed in a way that allows the active contour to emphasize particular aspects of the shape to be reconstructed. This formulation overcomes the limitation of previous statistical shape models [3], in which larger features of a shape dominate over relatively smaller, yet important features, merely because their large size influences the measures of shape variability.

(b) The degree of similarity between a particular snake configuration and the model is not merely an Euclidean distance in the 2D or 3D space, but rather the distance between their corresponding attribute vectors.

(c) The snake deformation is achieved by employing a hierarchy of transformations of a varying degree of smoothness, in order to adapt the initial model to the underlying object in the image. A key component of this deformation mechanism is that it doesn't deform snake points individually, but it deforms segments of the snake at a time, helping the snake avoid local minima.

\section{Geometric description of a shape}

In this section, we describe the affine-invariant attribute vectors $[7,8]$ as a means to characterize shape properties around each point of an active contour, which will be later used to design an energy term of the snake model. The attribute vector holds parameters that describe the shape characteristics of the model contour around each point. In this paper, the components of the attribute vector are the areas of triangles shown in figure 
1. For example, the area of the triangle $V_{i-1} V_{i} V_{i+1}$ approximates the curvature around $V_{i}$. Areas of larger triangles represent more global properties of the contour. It is not hard to see that the attribute vector corresponding to $V_{i}$ is different from attribute vectors of other points along the contour. We use this fact in the deformation process to preserve the configuration of the snake.

Let's define a snake $C_{\text {snake }}$ by an ordered set of points, commonly called snaxels, $\left\{V_{i}=\left(x_{i}, y_{i}\right) \mid i=1,2, \ldots, N\right\}$. For the $i$ th snaxel $\left(x_{i}, y_{i}\right)$, its corresponding attribute vector can be defined as the areas of $R$ triangles:

$F_{i}=\left[\begin{array}{ll}f_{i, 1} & f_{i, 2} \ldots . . f_{i, R}\end{array}\right]^{T}$
where $f_{i, v s}=\frac{1}{2}\left|\begin{array}{ccc}x_{[i-v s]} & x_{i} & x_{[i+v s]} \\ y_{[i-v s]} & y_{i} & y_{[i+v s]} \\ 1 & 1 & 1\end{array}\right|$ is the area of a triangle formed by $\left(x_{[i-v s]}, y_{[i-v s]}\right),\left(x_{i}, y_{i}\right)$, and $\left(x_{[i+v s]}, y_{[i+v s]}\right)$ (see figure 1). Notice that $[i+v s]=[i+v s+N] \% N$. The size of $R$ effectively determines the sampling resolution of the curve for our representation. Each attribute vector $F_{i}$ can be made exactly affine-invariant by the following normalization:

$$
\hat{F}_{i}=\frac{F_{i}}{\sum_{i=1}^{N} \sum_{v s=1}^{R}\left|f_{i, v s}\right|},
$$

where $\quad \hat{F}_{i}=\left[\begin{array}{ll}\hat{f}_{i, 1} & \hat{f}_{i, 2} \ldots . \hat{f}_{i, R}\end{array}\right]^{T}$. Thus, the shape information of the curve $C_{\text {snake }}$ can be described by a set of affine-invariant attribute vectors, $\left\{\hat{F}_{i}, i=1,2, \ldots, N\right\}$.

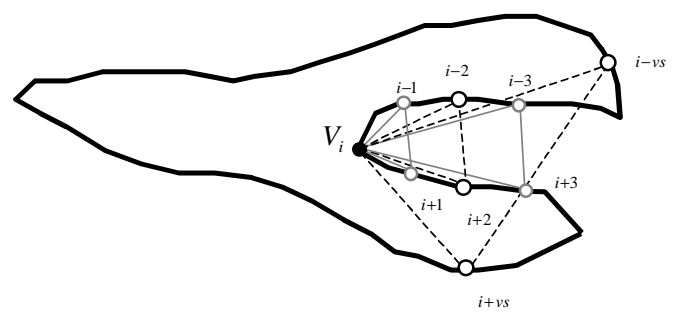

Fig. 1 Schematic representation of the concept of the "attribute vector" on the $i$-th snaxel. The area of a triangle, formed by $V_{i-v s}, V_{i}$, and $V_{i+v s}$, is used as the $v s$-th element of the $i$-th attribute vector. Here, $1 \leq v s \leq R$.

\section{Hierarchical snake deformation}

In this section, a hierarchical snake deformation mechanism is proposed. In this mechanism, the segments of the model seek the boundaries with the similar shape structure in an image edge map, and are not simply influenced by any nearby edge. This is achieved by an energy term in the snake formulation, which measures the degree of similarity between a particular snake configuration and the model, as reflected by the Euclidean distance between their corresponding attribute vectors. This mechanism is described in Section 3.1. In Section 3.2, a hierarchy of global and local affine transformations is designed as a deformation strategy for the snake. This deformation is very robust to local minima, since it deforms the segments of the snake, not individual points. Finally, in order to capture the finest details of the boundary of interest, a technique based on a local-curve fitting is presented in Section 3.3. In the following, the energy definition of our snake model is given. Then, the energy minimization approaches are discussed.

As customary, the total energy of the snake is defined as the weighted summation of several energy terms:

$$
E_{\text {snake }}=\sum_{i=1}^{N} \omega_{i} E_{i}=\sum_{i=1}^{N} \omega_{i}\left(E_{i}^{\text {model }}+E_{i}^{\text {data }}\right),
$$

where $\omega_{i}$ is a weighting parameter for the $i$-th snaxel. $E_{\text {snake }}$ is composed of two terms: $E_{i}^{\text {model }}$ and $E_{i}^{\text {data }}$, which are defined in detail in Section 3.1. The term $E_{i}^{\text {model }}$ defines the degree of similarity between the snake and the model. The term $E_{i}^{\text {data }}$ defines the external energy, aiming at deforming the snake towards a boundary in the image.

In the literature, many different approaches for minimizing the snake energy have been reported. In this paper, we will use the greedy algorithm [9] as an optimization technique, which has been found to be one of the most reliable, fast, and flexible local strategies.

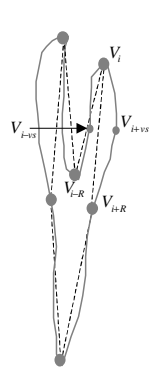

(a)

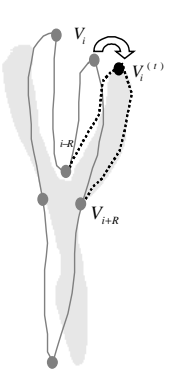

(b)

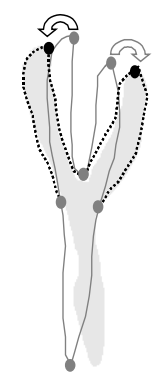

(c)

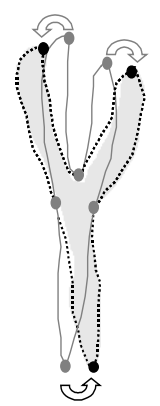

(d)
Fig. 2 The definition of the $i$ th snake segment and its affine transformation. The black arrows in (b-d) indicate the affine transformations of the current snake segments, while the grey arrows represent the affine transformations already completed.

\subsection{Snake energy definition}

According to the internal energy term of the standard snake, the motion of every snaxel $\left(V_{i}\right)$ is constrained only by its two nearest neighborhoods $V_{[i-1]}$ and $V_{[i+1]}$ at each iteration. This can lead to two unwanted situations. First, it can cause unrealistic deformations of the snake as individual points are pulled towards noisy or fragmented 
edges. Second, it does not preserve the geometric structure of the contour, i.e. it can dramatically change its attribute vectors.

Our model doesn't deform snake points individually, but it deforms segments of the snake at a time, helping the snake avoid local minima. It also restricts the deformation process to be relatively smooth. In order to achieve this, we require that, during its deformation, the snake should be able to preserve its geometric structure as reflected by the attribute vectors. Accordingly, we define the first term $E_{i}^{\text {model }}$ in equation (1) as the difference between the snake's attribute vector and the model's attribute vector, which is mathematically given in equation ( $2 \mathrm{a})$. Here, our model is an average sample.

$$
E_{i}^{\text {model }}=\sum_{v s=1}^{R} \delta_{v s}\left(\hat{f}_{i, v s}^{\text {Snk }}-\hat{f}_{i, v s}^{M d l}\right)^{2},
$$

where $\hat{f}_{i, v s}^{\text {Snk }}$ and $\hat{f}_{i, v s}^{\text {Mdl }}$ are the normalized attribute elements (areas of triangles) respectively for the snake and the model. The parameter $\delta_{|v s|}$ is the importance degree of the $v s$-th attribute element $\hat{f}_{i, v s}^{s n k}$ in the segment under consideration. The $i$ th snake segment is defined as the set of $2 R+1$ points, $\left\{V_{[i+v s]} \mid,-R \leq v s \leq R\right\}$, around the $i$ th snaxel $V_{i} \cdot R$ is the number of geometric attributes, and also used for determining the length of snake segment. See figure 2(a) for example.

The data energy term is usually designed to move the snake towards a boundary of interest in the image. Accordingly, for every snaxel $V_{i}$, we can require that in the position of $V_{i}$, the magnitude of image gradient should be high, and the direction of image gradient should be similar to the normal vector of the snake. Since we suggest deforming the whole snake segment around each snaxel $V_{i}$ at a time, the design of the data energy term $E_{i}^{\text {data }}$ for the $i$-th snake segment results by summing individual terms along the $i$-th snake segment:

$$
E_{i}^{\mathrm{data}}=\sum_{v s=-R}^{R} \delta_{|v s|}\left(1-\left|\nabla I\left(V_{[i+v s]}\right)\right| \cdot\left|\vec{h}\left(V_{[i+v s]}\right) \cdot \vec{n}\left(V_{[i+v s]}\right)\right|\right)
$$

where $\left|\nabla I\left(V_{i}\right)\right|$, valued between 0 and 1 , is the normalized magnitude of the gradient on the snaxel $V_{i} ; \vec{h}\left(V_{i}\right)$ is the direction of the gradient; $\vec{n}\left(V_{i}\right)$ is the normal vector of the snake in a given snaxel $V_{i}$, directed towards the snake interior.

From equations (1), (2a) and (2b), the whole energy function of the snake can be obtained as follows:

$$
\begin{aligned}
E_{\text {snake }}= & \sum_{i=1}^{N} \omega_{i}\left[\sum_{v s=1}^{R} \delta_{v s}\left(\hat{f}_{i, v s}^{S n k}-\hat{f}_{i, v s}^{M d l}\right)^{2}\right. \\
& \left.+\sum_{v s=-R}^{R} \delta_{|v s|}\left(1-\left|\nabla I\left(V_{[i+v s]}\right)\right| \cdot\left|\vec{n}\left(V_{[i+v s]}\right) \cdot \vec{h}\left(V_{[i+v s]}\right)\right|\right)\right]
\end{aligned}
$$

\subsection{Snake deformation mechanism}

We now describe a greedy deformation algorithm for the minimization of the snake energy function in equation (3). We have defined the energy terms $E_{i}^{\text {model }}$ and $E_{i}^{\text {data }}$, and suggested considering the deformation of a snake segment as whole. In particular, in each iteration that represents a deformation of the snake, a segment of $(2 R+1)$ points is deformed by an affine transformation. The energy function in equation (3) is evaluated by integrating along this snake segment, and it is optimized via a greedy search. The reason for transforming snake segments by affine transformations is because the value of the energy term $E_{i}^{\text {model }}$ remains unchanged under affine transformation of the $i$-th snake segment since the attribute vector is affine-invariant. Accordingly, the new configuration of a particular segment can be determined directly by minimizing an energy term $E_{i}^{\text {model }}$ that is found by integrating the individual energy terms along the snake segment. This procedure, which greatly helps the snake avoid local minima, is defined next.

\subsubsection{Affine transformation of the snake segment}

The affine-transformation of a snake segment can be described by the affine transformation of the triangle defined by snaxels $V_{[i+R]}, V_{[i-R]}$ and $V_{i}$. Let's assume that a tentative position of the $i$ th point in the greedy algorithm is $V_{i}^{(t)}$. Then, this tentative selection of the $i$ th point will lead to the shape change of the related triangle, from $\Delta_{V_{[i-R]} V_{i} V_{[i+R]}}$ to $\Delta_{V_{[i-R]} V_{i}^{(t)} V_{[i+R]}}$ (figure 2b). The related deformation procedure can be viewed as an affine transformation $A$, where $A$ is an affine-transformation matrix.

Suppose that all the snaxels on the $i$ th segment move with the three vertices $\left(V_{[i-R]}, V_{i}\right.$ and $\left.V_{[i+R]}\right)$ according to the same affine transformation $A$. Then, the value of the energy term $E_{i}^{\text {model }}$ will remain unchanged because of the affine-invariant nature of $E_{i}^{\text {model }}$. In the greedy algorithm, we only need to examine the match between the currently affine-deformed snaxels and the object boundaries by minimizing $E_{i}^{\text {Data }}$.

A mathematical expression of the above affinetransformation strategy can be detailed here, by determining the affine transform $A$ first and then transforming the involved snaxels by the matrix $A$. The matrix $A$ is given by

$$
A=\left[\begin{array}{ccc}
x_{[i-R]} & x_{i}^{(t)} & x_{[i+R]} \\
y_{[i-R]} & y_{i}^{(t)} & y_{[i+R]} \\
1 & 1 & 1
\end{array}\right] \cdot\left[\begin{array}{ccc}
x_{[i-R]} & x_{i} & x_{[i+R]} \\
y_{[i-R]} & y_{i} & y_{[i+R]} \\
1 & 1 & 1
\end{array}\right]^{-1} .
$$


Figure 2(b) shows the deformation of the $i$ th snake segment, while the deformations for the upper-left and the lower snake segments can be found respectively in figure 2(c) and figure 2(d).

\subsubsection{Hierarchical deformation strategy}

In the definition of the total snake energy function $E_{\text {snake }}$, the parameter $R$ determines the length, $(2 R+1)$, of the snake segment. The value of $R$ is typically large in the initial iterations, and gradually reduces to 1 . The whole hierarchical deformation strategy suggested in Section 3.2 can be summarized as follows:

(1) Use a large value of $R$ to determine the best affinetransformed configuration of the snake segment around $V_{i}$. At this point, the number of snake segments that are considered is small, and the search area is large.

(2) Reduce the value of $R$ to update the affinetransformed configuration of the snake segment around $V_{i}$. The number of snake segments that are considered becomes larger, while the search area becomes smaller.

(3) Finally, set $R$ equal to 1 . Update the affinetransformed snake segment around $V_{i}$. At this level, all snaxels are considered.

\subsection{Fine deformation by local-curve fitting}

The deformation mechanism we described in Section 3.2 is very robust, but often at the expense of smoothing out the very fine details of the boundary. In order to achieve better conformity to the shape of an object, we employ a curve fitting procedure that is described next. This procedure constitutes the final fine-tuning step of our algorithm.

In particular, we select the position $V_{i}^{\text {fine }}$ of the snaxel $V_{i}$ from the non-zero Canny edge points in the neighborhood of the snaxel $V_{i}$. This position $V_{i}^{\text {fine }}$ is the one for which the snake segment $\overrightarrow{V_{[i-1]} V_{i}^{\text {fine }} V_{[i+1]}}$ and the locally-connected image edge segment are in best agreement. The maximum-compression process employed by the Canny edge detector results in edge maps with relatively few non-zero points. Therefore, the search for edges in the neighborhood of a snaxel can be performed fast. In figure 3 , the neighborhood of the snaxel $V_{i}$ is drawn as a grey dotted circle. The snake segment $\overrightarrow{V_{[i-1]} V_{i} V_{[i+1]}}$ is shown as a thick grey curve, connecting three consecutive snaxels $V_{[i-1]}, V_{i}$ and $V_{[i+1]}$. The snake segment $\overrightarrow{V_{[i-1]} V_{i}^{\text {fine }} V_{[i+1]}}$ resulting from a tentative placement of the $i$-th snaxel at $V_{i}^{\text {fine }}$ is shown as a thin black curve. The locally-connected image edge segment, with the fine point $V_{i}^{\text {fine }}$ on it, is shown as a thick black curve. Notice here, for different selected position of $V_{i}^{\text {fine }}$, the locally-connected image edge segments might be different. In figure 3 , the value $D$ is used to represent the size of the neighborhood of the local snake curve $\overrightarrow{V_{[i-1]} V_{i}^{\text {fine }} V_{[i+1]}}$, which is enclosed by the dotted lines. The degree of similarity between the extracted edge segment $\operatorname{Seg}\left(V_{i}^{\text {fine }}\right)$ and the local snake $\overrightarrow{V_{[i-1]} V_{i}^{\text {fine }} V_{[i+1]}}$ is defined as the total length of the extracted edge segment contained in the neighborhood of $\overrightarrow{V_{[i-1]} V_{i}^{\text {fine }} V_{[i+1]}}: \operatorname{Length}\left(\operatorname{Seg}\left(V_{i}^{\text {fine }}\right), \overrightarrow{V_{[i-1]} V_{i}^{\text {fine }} V_{[i+1]}}\right)$.

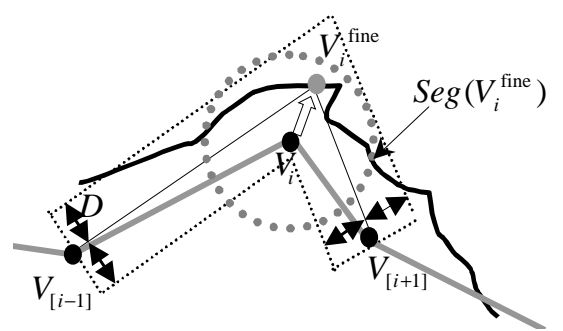

Fig. 3 Local-curve fitting. The fitting degree between the extracted edge segment $\operatorname{Seg}\left(V_{i}^{\text {fine }}\right)$ and the local snake curve $\overrightarrow{V_{[i-1]} V_{i}^{\text {fine }} V_{[i+1]}}$ is defined as the total length of the extracted edge segment contained in the neighborhood of the local curve $\overrightarrow{V_{[i-1]} V_{i}^{\text {fine }} V_{[i+1]}}$. See the text for details.

The procedure of curve fitting, which determines the final position of the snaxel $V_{i}$, is summarized next:

(a) Suppose $V_{i}^{\text {fine }}$ is the position of a selected non-zero Canny edge point in the neighborhood of the snaxel $V_{i}$

(b) Regard this Canny edge point as the seed point, and then track the connected edge segment, $\operatorname{Seg}\left(V_{i}^{\text {fine }}\right)$, of this seed point from Canny edge map. Different $V_{i}^{\text {fine }}$ probably extracts different image edge segment.

(c) Calculate the degree of similarity, $\operatorname{Length}\left(\operatorname{Seg}\left(V_{i}^{\mathrm{fine}}\right), \overrightarrow{V_{[i-1]} V_{i}^{\text {fine }} V_{[i+1]}}\right), \quad$ between the extracted edge segment $\operatorname{Seg}\left(V_{i}^{\text {fine }}\right)$ and the snake curve $\overrightarrow{V_{[i-1]} V_{i}^{\text {fine }} V_{[i+1]}}$.

(d) The final fine position of $V_{i}$ is determined by the best fine position $V_{i}^{\text {fine }}$, which maximize the fitting degree $\operatorname{Length}\left(\operatorname{Seg}\left(V_{i}^{\mathrm{fine}}\right), \overrightarrow{V_{[i-1]} V_{i}^{\text {fine }} V_{[i+1]}}\right)$ in the whole neighborhood of the snaxel $V_{i}$. 


\section{Adaptive-focus statistical model}

In this section, an adaptive-focus statistical model is suggested, which allows the deformable model to emphasize particular aspects of the shape to be reconstructed. This formulation overcomes the limitation of other statistical shape models [3], in which larger features of a shape dominate over relatively smaller, yet important features, merely because their large size influences the measures of shape variability. Moreover, our confidence in certain features or parts of features might be relatively higher compared to the rest of the shape. Therefore, we might want to focus, at first, on those reliable parts of the shape, and subsequently to shift the focus to other parts as they become closer to their respective targets and therefore more reliable. For example, in segmenting basal ganglia and ventricular boundaries from brain images (figure 6), a priori knowledge is available that reflects our level of confidence in certain parts of the shape under consideration. The ventricle usually has a stronger boundary in the brain image and thus is easier to find. Therefore, we can initially focus on the ventricle, by assigning a higher weight to it. As the ventricular boundaries are detected, our confidence for the neighboring basal ganglia becomes much higher, because of their relatively fixed location relative to the ventricles.

It should be indicated here that a different weighting matrix employed leads to a different subspace spanned by a selected set of eigenvectors derived from the training set.

The details of our formulation are described in Sections 4.1 and 4.2 .

\subsection{Shape alignment and weighting strategy}

Given a set of training samples, we first need to align them into a common coordinate frame, before performing the weighting strategy and the statistics on them. The alignment technique used in this paper is the affineinvariant alignment method [7].

In the alignment procedure, one training sample is selected as a standard shape and others are transformed to best fit it. Suppose that the affine transform matrix relating one training shape $\left\{V_{i} \mid i=1,2, \ldots, N\right\}$ and the standard shape is $A^{\text {align }}$. Then the coordinates of the point $V_{i}=\left(x_{i}, y_{i}\right)$ after shape alignment become

$$
\left[\begin{array}{c}
x_{i}^{\text {align }} \\
y_{i}^{\text {align }} \\
1
\end{array}\right]=A^{\text {align }}\left[\begin{array}{c}
x_{i} \\
y_{i} \\
1
\end{array}\right] \text {. }
$$

For simplicity in the notation, the aligned shape $\left\{\left(x_{i}^{\text {align }}, y_{i}^{\text {align }}\right) \mid i=1,2, \ldots, N\right\}$ can be stacked and represented by a $2 N$-element column vector $P$ :

$$
P=\left[x_{1}^{\text {align }}, y_{1}^{\text {align }}, \ldots, x_{N}^{\text {align }}, y_{N}^{\text {align }}\right]^{r} .
$$

Once all the training samples have been aligned, the statistical model can be obtained from the set of the aligned shapes $\{P\}$.

Before introducing our adaptive focus model, we note that active shape models are typically based on statistics on the coordinate vector $P$. However, a much broader class of active shape models can be obtained by applying a linear transformation to the vector $P$, by multiplying it by a matrix $W^{F e a}$. The purpose of $W^{F e a}$ is to extract certain features from the vector $P$. By specially designing $W^{F e a}$, we can obtain Fourier coefficients, Wavelet coefficients or Gabor coefficients. Moreover, a weighting matrix $W^{\text {Weig }}$ can be applied, enhancing certain aspects of the shape. If $W^{F e a}=I$ and $W^{\text {Weig }}$ is a diagonal matrix, we obtain a model that weights different points of the snake differently. By adapting $W^{\text {Weig }}$ during the snake's deformation, we develop an adaptive-focus model, which is able to zoom in and out of individual parts of an object. We continue our development by introducing the feature vector

$$
S=W \cdot P,
$$

where $W=W^{\text {Weig }} \cdot W^{\mathrm{Fea}}$. Assume that $M$ is the number of the features obtained by the feature extraction matrix $W^{F e a}$. Then, the size of the matrix $W^{F e a}$ should be $M \times 2 N$, the size of $W^{\text {Weig }} M \times M$, and the size of matrix $W M \times 2 N$. The column vector $S$ has $M$ elements.

\subsection{Refinement of the snake contour}

In this section, we describe how we utilize the statitical shape information derived from the training set, in order to constrain the deformable model to be in the space of allowable (or likely) configurations.

After aligning all the training samples into a common coordinate frame, we can calculate both the average feature vector $S_{\text {mean }}$ and the covariance matrix $(M \times M)$. $M$ eigenvectors of the covariance matrix can be calculated and ranked by the sizes of their corresponding eigenvalues. From the statistical theory, the $M_{\text {Select }}$ eigenvectors corresponding to the $M_{\text {Select }}$ highest eigenvalues can be selected as the basis of the shape subspace of the training samples. Note that, for a different transformation matrix $W$, there will be different covariance matrix and also a different shape subspace spanned by the $M_{\text {Select }}$ selected eigenvectors. For simplicity in the notation, we can stack these $M_{\text {Select }}$ eigenvectors as a $M \times M_{\text {Select }}$-sized matrix $H$, where each column is one of eigenvectors.

After some algebraic manipulations, we arrive at the following formula for fitting the model instance $P^{\prime}$ to the point vector $P$ of the aligned snake shape: 


$$
P^{\prime}=T_{1} \cdot T_{2} \cdot P+T_{3},
$$

where $\quad T_{1}=W^{-1} \cdot H, \quad T_{2}=H^{T} \cdot W \quad$ and $T_{3}=\left(W^{-1}-W^{-1} \cdot H \cdot H^{T}\right) S_{\text {mean }}$. The sizes of the matrices $T_{1}, T_{2}$ and $T_{3}$ are, respectively, $2 N \times M_{\text {Select }}, M_{\text {Select }} \times 2 N$ and $2 N \times 1$. Once obtaining the best model instance $P^{\prime}$,

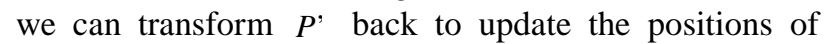
snaxels in the current snake just by using the inverse matrix of $A^{\text {align }}$.

\subsection{An algorithm of statistical snake refinement}

The algorithm for refining the set of snake snaxels by the statistical model is summarized as:

(1) Align the snake contour $\left\{V_{i} \mid i=1,2, \ldots, N\right\}$ with the selected standard shape by using the alignment matrix $A^{\text {align }}$, which is calculated by the alignment method. Afterwards, stack the aligned snake as a column point vector $P$,

$$
P=\left[x_{1}^{\text {align }}, y_{1}^{\text {align }}, \ldots, x_{N}^{\text {align }}, y_{N}^{\text {align }}\right]^{T} .
$$

(2) Use equation (4) to correct the point vector $P$ into a new vector $P^{\prime}$.

(3) Update snake contour by transforming $P^{\prime}$ back to the original coordinate space of the snake contour via the inverse matrix of $A^{\text {align }}$.

\section{Experiments}

The complete algorithm for our method is summarized in Section 5.1. To evaluate our algorithm, two sets of experiments are presented here. The first set of experiments (Section 5.2) demonstrates the performance of our whole model in the case of the identical weights in the matrix $W^{\text {Weig }}$; this corresponds to the non-adaptive focus model. The second set of experiments (Section 5.3) shows the performances of our adaptive-focus model. In where, we focus on demonstrating the effect of our weighting strategy.

In all experiments, the initialization of the snake is provided by the user. The search time of our algorithm depends on the initialization of snake and the complexity of the studied image. It ranges from 1 second to several seconds in an SGI OCTANE workstation.

\subsection{Complete algorithm}

The complete algorithm is as follows:

(1) Select a small number of snake segments (or snaxels). For each snake segment, its length and the corresponding search area are both larger initially. The length of each snake segment is $(2 R+1)$, where $R$ is assigned a larger integer value initially.
(2) For every snake segment in the set of selected snake segments, determine its best affine-transformed configuration by minimizing its external energy term $E_{i}^{\text {Data }}$. Here, the local affine-transformation technique is employed to locally deform the snake segment that is considered. (Details in Section 3.2)

(3) Align the current snake configuration with the standard model contour by using the affinetransformation matrix $A^{\text {align }}$. Then, stack the aligned snake as a point vector $P$,

$$
P=\left[x_{1}^{\text {align }}, y_{1}^{\text {align }}, \ldots, x_{N}^{\text {align }}, y_{N}^{\text {align }}\right]^{T} .(\text { Section } 4.1)
$$

(4) Map the point vector $P$ into the new vector $P^{\prime}$ using the adaptive-focus statistical model, $P^{\prime}=T_{1} \cdot T_{2} \cdot P+T_{3}$. Then, transform $P^{\prime}$ back into the original coordinate space of the snake contour via the inverse matrix of $A^{\text {align }}$, and update the snake.

(5) Increase the number of snake segments to be considered, and decrease both the length of snake segment and the size of search area of every selected snake segment. If the length of the snake segment is equal to 3 , i.e. $R=1$, then go to step (6); otherwise, repeat steps (2-5).

(6) Use the fine deformation strategy to refine the configuration of the snake to the object boundary. (Section 3.3)

\subsection{The case of equal weights}

In this set of experiments, we applied our method, with the equal weights $\left(W^{\text {Weig }}=I\right.$ ), not adaptive, for segmenting the ventricles from the MRI brain images. Some typical training examples, selected from the set of 118 , are shown in figure 4 . In figure 4 , the grey contour represents the standard shape of the ventricle. The black contours are the individual shapes, aligned to the standard shape.

A key property of the proposed method is its tendency to maintain the geometric shape of the snake model during the shape deformation procedure. Figure 5 qualitatively compares the performances of our method, the standard snake, and standard active shape model (ASM) in detecting ventricles from the MRI brain images (256x256). Initializations for three different MRI images are provided in figures $5(\mathrm{a} 1, \mathrm{a} 2, \mathrm{a} 3)$, where crosses ' + ' are used as labels in order to track the deformation of individual points. Using the standard snake, the final results were shown in figures $5(\mathrm{~b} 1, \mathrm{~b} 2, \mathrm{~b} 3)$. In addition to being trapped by erroneous edges, the standard snake did not preserve any anatomical homology during the deformation, as reflected by the positions of crosses after the snake deformation. ASM is able to preserve the shape of the model in the procedure of deformation, however, sometimes owing to local minimum problem its final results $(\mathrm{c} 1, \mathrm{c} 2, \mathrm{c} 3)$ are still unsatisfactory. On the contrary, 
our method maintains the geometric shape during the detection procedure, and thus gives good results, as shown in figures $5(\mathrm{~d} 1, \mathrm{~d} 2, \mathrm{~d} 3)$. Table 1 gives the quantitative comparisons of our algorithm with the standard snake and ASM, based on the example results given in figure 5 . In table 1 , the average distance of the final contour from the contour marked by experts is given, at units of pixel. Our algorithm has the least average distances (1.3 1.7 pixels) for all three examples, compared to the standard snake and ASM. Also, the maximal distance between the final contour and the contour marked by experts was calculated. Our algorithm still has the least maximal distances (4.1 4.6 pixels), compared to the other two methods.

\subsection{The case of different weights}

We have suggested using different weights for different components in our adaptive-focus statistical model. Experiment using this model on MR images is given next.

The goal in this experiment was to segment the basal ganglia and ventricular boundaries from MR brain images. In this application, the weights of the adaptivefocus statistical model can be determined by the degrees of our confidence in the components of the shape under consideration. See figure 6 for example. We can assign larger weights to the landmarks of the ventricles just because the ventricles usually have a stronger boundary that is easier to locate. Smaller weights are assigned to the landmarks of the basal ganglia, which have relatively unreliable and fuzzy boundaries often confounded by adjacent cortical edges. Figure 6(b) shows a 100-point model, derived from a set of 10 basal ganglia and ventricular boundaries, on the original testing image (256x256). Training samples are shown in figure 6(a). By using our adaptive-focus deformable model, a satisfactory result is obtained and shown in figure 6(c). It can be observed that the deformation from the initialization to the final result is large (particularly for the lower part of this object), and our model is robust against the attraction from the boundaries of cortex because of using the suggested weighting strategy in adaptive-focus statistical model.

\section{Conclusion and future work}

In this paper, we have proposed an adaptive-focus deformable model for segmenting 2D deformable objects from images. The source codes for our adaptive-focus deformable model are freely available from the site, http://pandora.cbmv.jhu.edu/ dgshen/SnakeCode.htm.

Several extensions of our methodology are possible. Currently, the boundary points are used to represent the object shape and also the snake segment. For speeding up our technique, the snake segment can be expressed as a
B-spline. Then, the deformation of the snake segment can be viewed as a B-snake under affine-transformation constraint [10].

Extension to our technique for 3D is also possible. In this paper, we particularly focused on designing the 2D version of our deformable model. We are now extending our $2 \mathrm{D}$ technique to $3 \mathrm{D}$ by redefining the geometric features and the deformation strategies.

\section{Reference:}

1. T. McInerney and D. Terzopoulos, "Deformable models in medical image analysis: a survey", Medical Image Analysis, 1(2): 91-108, 1996.

2. C. Davatzikos, "Spatial Transformation and Registration of Brain Images Using Elastically Deformable Models", Comp. Vis. And Image Understanding, Special Issue on Medical Imaging, 66(2): 207-222, May 1997.

3. T.F. Cootes, D. Cooper, C.J. Taylor and J. Graham, "Active shape models-their training and application", Computer Vision and Image Understanding, Vol. 61, No. 1, pp. 38-59, Jan. 1995.

4. G. Szekely, A. Kelemen, C. Brechbuhler, G. Gerig, "Segmentation of 2-D and 3-D objects from MRI volume data using constrained elastic deformations of flexible Fourier contour and surface models", Medical Image Analysis, 1(1): 19-34, 1996.

5. L.H. Staib and J.S. Duncan, "Boundary finding with parametrically deformable models", IEEE Trans. on PAMI, 14(11):1061-1075, 1992.

6. Julia A. Schnabel, Simon R. Arridge, "Active shape focusing", Image And Vision Computing, (17):5-6, pp.419428, 1999.

7. Dinggang Shen, W. H. Wong and Horace H. S. Ip, “Affine invariant image retrieval by correspondence matching of shapes", Image and Vision Computing, 17(7): 489-499, May, 1999

8. Horace H. S. Ip, Dinggang Shen, "An affine-invariant active contour model (AI-snake) for model-based segmentation", Image and Vision computing, 16(2): 135146, 1998.

9. D. J. Williams and M. Shah, "A fast algorithm for active contours and curvature estimation", Computer Vision, Graphics, Image Processing, 55:14-26, 1992.

10. Yue Wang, Eam Khwang Teoh, and Dinggang Shen, "Lane detection using B-snake", IEEE International Conference on Information, Intelligence and Systems, Washington, DC, Nov. 1-3, 1999.

11. T.F. Cootes, C.J. Taylor, A. Lanitis, "Active shape models: evaluation of a multi-resolution method for improving image search", in Proc. British Machine Vision Conference, pp.327-336, 1994.

12. M. Chen, T. Kanade, D. Pomerleau, J. Schneider, "3-D deformable registration of medical images using a statistical atlas", MICCAI, Sept. 1999. 

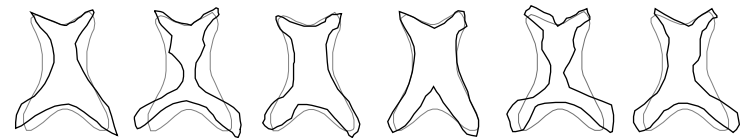

Fig. 4 Some typical training samples aligned and used in our statistical model. The grey contour represents the standard shape, while the black ones denote the individual shapes aligned to the standard shape via affine transformations. There are totally 118 samples in the training set of ventricles.

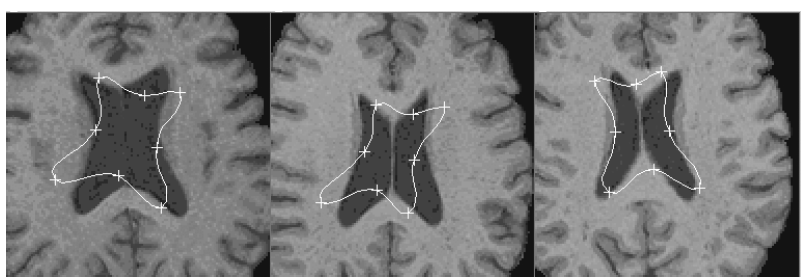

(a1) (a2) (a3)

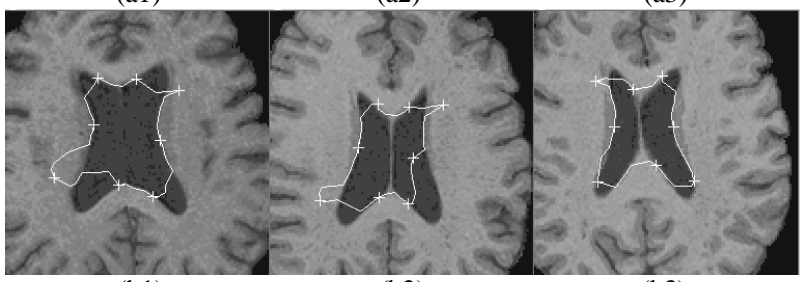

(b1)

(b2) (b3)

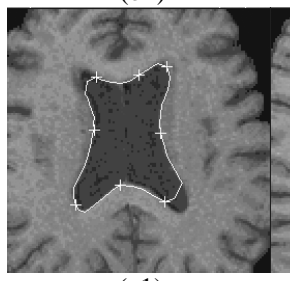

(c1)

(c2)

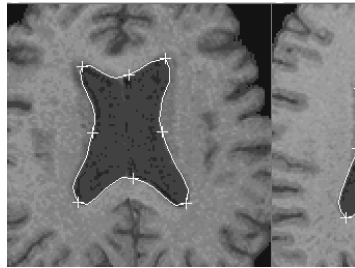

(d1)

(d2)

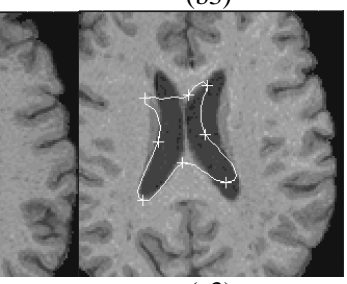

(c3)

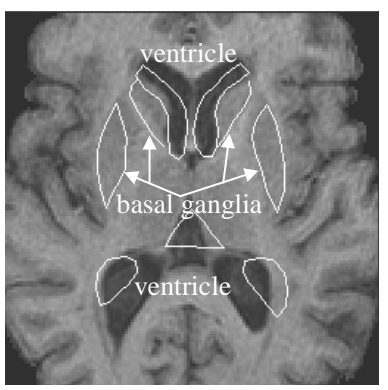

(b)

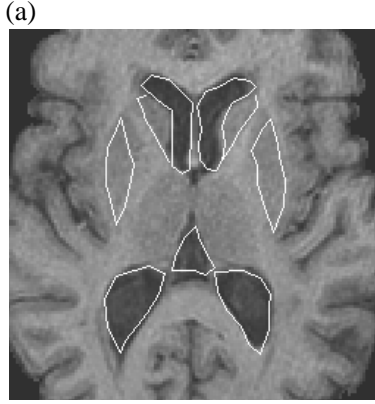

(c)

Fig. 6 Example using spatially variable weighting. Here, larger weights are assigned to ventricles, because they have a stronger boundary and are easier to locate. Also, smaller weights are assigned to the landmarks of basal ganglia, because basal ganglia have unreliable and fuzzy boundaries often confused with adjacent cortical edges. The deformation is driven primarily by the ventricular boundaries of the model. As those get close to their final positions, the adjacent basal ganglia boundaries also get close to their final positions, and therefore become more reliable features to drive the deformation. (a) Training samples (256x256); (b) a 100-point model derived from a set of samples is placed on the original testing image; (c) final result.

Fig. 5 Qualitative comparisons of our algorithm with the standard snake and ASM. (a1,a2,a3) the manual initializations, $(\mathrm{b} 1, \mathrm{~b} 2, \mathrm{~b} 3)$ the results of the standard snake, $(\mathrm{c} 1, \mathrm{c} 2, \mathrm{c} 3)$ the results of ASM, and $(\mathrm{d} 1, \mathrm{~d} 2, \mathrm{~d} 3)$ the results from our model.

Table 1 Quantitative comparisons of our algorithm with the standard snake and ASM, using the results already given in figure 5.

\begin{tabular}{|c|c|c|c|c|c|c|}
\hline & \multicolumn{2}{|c|}{ Fig.5(a1) } & \multicolumn{2}{c|}{ Fig.5(a2) } & \multicolumn{2}{c|}{ Fig.5(a3) } \\
\cline { 2 - 7 } & average dist. & max dist. & average dist. & max dist. & average dist. & Max dist. \\
\hline Standard snake & 6.9 & 28.3 & 6.8 & 29.5 & 3.3 & 18.4 \\
\hline ASM & 2.5 & 18.0 & 2.8 & 11.7 & 5.9 & 22.8 \\
\hline Our algorithm & $\mathbf{1 . 3}$ & $\mathbf{4 . 1}$ & $\mathbf{1 . 2}$ & $\mathbf{4 . 0}$ & $\mathbf{1 . 7}$ & $\mathbf{4 . 6}$ \\
\hline
\end{tabular}

